\title{
Measurement of tidal breathing flows in infants using impedance pneumography
}

\author{
Leo Pekka Malmberg ${ }^{1}$, Ville-Pekka Seppä, Anne Kotaniemi-Syrjänen ${ }^{1}$, \\ Kristiina Malmström ${ }^{1}$, Merja Kajosaari ${ }^{3}$, Anna S. Pelkonen ${ }^{1}$, Jari Viik ${ }^{2}$ and \\ Mika J. Mäkelä ${ }^{1}$
}

Affiliations: 'Skin and Allergy Hospital, University of Helsinki and Helsinki University Hospital, Helsinki, Finland. ${ }^{2}$ Dept of Electronics and Communications Engineering, Tampere University of Technology, BioMediTech, Tampere, Finland. ${ }^{3}$ Children's Hospital, University of Helsinki and Helsinki University Hospital, Helsinki, Finland.

Correspondence: Leo Pekka Malmberg, Dept of Allergology, Helsinki University Hospital, PO Box 160, 00029 HUS, Helsinki, Finland. E-mail: pekka.malmbergahus.fi

@ERSpublications

Impedance pneumography is a novel noninvasive option for tidal flow profile and lung function monitoring in infants http://ow.ly/7RId305Oj80

Cite this article as: Malmberg LP, Seppä V-P, Kotaniemi-Syrjänen A, et al. Measurement of tidal breathing flows in infants using impedance pneumography. Eur Respir J 2017; 49: 1600926 [https://doi.org/10.1183/ 13993003.00926-2016].

ABSTRACT Tidal breathing flow volume (TBFV) profiles have been used to characterise altered lung function. Impedance pneumography (IP) is a novel option for assessing TBFV curves noninvasively. The aim of this study was to extend the application of IP for infants and to estimate the agreement between IP and direct pneumotachograph (PNT) measurements in assessing tidal airflow and flow-derived indices.

Tidal flow profiles were recorded for 1 min simultaneously with PNT and uncalibrated IP at baseline in 44 symptomatic infants, and after methacholine-induced bronchoconstriction in a subgroup $(\mathrm{n}=20)$.

The agreement expressed as the mean deviation from linearity ranged between 3.9 and $4.3 \%$ of tidal peak inspiratory flow, but was associated with specific airway conductance $(\mathrm{p}=0.002)$ and maximal flow at functional residual capacity $\left(V^{\prime} \operatorname{maxFRC}\right)(\mathrm{p}=0.004)$ at baseline. Acute bronchoconstriction induced by methacholine did not significantly affect the agreement of IP with PNT. TBFV indices derived from IP were slightly underestimated compared to PNT, but were equally well repeatable and associated with baseline $V^{\prime} \operatorname{maxFRC}(\mathrm{p}=0.012$ and $\mathrm{p}=0.013$, respectively).

TBFV profiles were consistent between IP and PNT in most infants, but the agreement was affected by reduced lung function. TBFV parameters were not interchangeable between IP and PNT, but had a similar association with lung function in infants.

This article has supplementary material available from erj.ersjournals.com

Received: May 092016 | Accepted after revision: Oct 292016

Support statement: The study was supported by The Finnish Medical Foundation, Foundation for Pediatric Research, Foundation for Allergy Research, Nummela Sanatorium Foundation and Helsinki University Hospital Research Funds. Funding information for this article has been deposited with the Open Funder Registry.

Conflict of interest: Disclosures can be found alongside this article at erj.ersjournals.com

Copyright @ERS 2017 


\section{Introduction}

Advances in infant lung function testing have improved the understanding of normal lung development and pathophysiology related to early lung diseases. Most techniques suited for infant lung function testing are laborious and require a considerable amount of expertise. One of the most convenient methods includes the analysis of tidal breathing flow volume (TBFV) profiles [1]. This technique has been used to characterise lung function in infants with cystic fibrosis [2], chronic lung disease due to prematurity [3] and wheezing disorders $[4,5]$, and in prospective birth cohorts that have shown TBFV indices to be associated with asthma later in life [6].

The analysis of TBFV indices requires accurate recording of tidal airflow profiles, which has been accomplished primarily in a laboratory setting directly from the mouth using a pneumotachograph (PNT) for limited periods [7]. Techniques that sensor chest wall movements, such as respiratory inductive plethysmography, have been successfully used to assess rhythmic and timing properties of breathing patterns in infants, but are less technically suited for characterising the flow-volume shape [8].

Impedance pneumography (IP) is a method for measuring changes in the thoracic electrical impedance through skin electrodes, which varies as a function of lung aeration, i.e. breathing. Over the past decades there have been several attempts to use impedance for regional or whole-lung tidal volume measurements $[9,10]$, but thus far only respiratory rate measurement in intensive care settings has gained wider clinical acceptance. However, recent technical advancements in IP signal processing [11] and electrode placement [12] have enabled IP to be used for noninvasive estimation of tidal airflow profiles. High agreement has been demonstrated in relative flow signal and TBFV indices between IP and direct PNT in young children, even during induced bronchoconstriction [13]. Moreover, in overnight recordings at home, IP was found to be feasible for quantifying nocturnal TBFV variability in young children with lower respiratory symptoms [14]. There are currently no previous data using IP in tidal flow measurement in infants with or without airway obstruction.

The primary objective of this validation study was to evaluate the agreement between uncalibrated IP and a direct PNT recording of tidal flow and TBFV indices in infants with recurrent airway symptoms. The agreement was assessed at baseline conditions and in a subsample of infants during methacholine-induced bronchoconstriction.

\section{Methods}

\section{Study subjects and design}

The study subjects included a total of 50 infants who had been referred to a tertiary centre due to troublesome dyspnoea, wheeze and/or cough, and who underwent infant lung function tests. Their demographic data including medication are shown in table 1 . Short-acting beta2-agonists were withheld for at least $12 \mathrm{~h}$ preceding the tests.

The design of the study included simultaneous recordings of tidal breathing using PNT and IP, lasting at least $60 \mathrm{~s}$, during sedation in a supine position. Tidal breathing was recorded at the baseline condition before (BL1) and after (BL2) measuring airway resistance and lung volumes with infant body plethysmography. In those infants who underwent the bronchial challenge test, the tidal breathing recordings were repeated during methacholine-induced bronchoconstriction (MIB), and after the inhalation of a bronchodilator (BRD).

The study was approved by the institutional paediatric ethics committee of Helsinki University Central Hospital (approval number 53/13/03/03/2012). The tests were conducted after written consent was received from the parents.

\section{Lung function measurements}

For tidal flow recordings, the flow was measured at the airway opening via a mask sealed with a rim of silicone putty, using a calibrated, heated, solid-state infant PNT (Babybody Masterscreen, Jaeger GmbH, Würzburg, Germany) with a dead space of $1.3 \mathrm{~mL}$ and accuracy of $\pm 3 \%$. After body temperature pressure saturated correction, data were digitised with a sampling frequency of $100 \mathrm{~Hz}$ and later oversampled to $256 \mathrm{~Hz}$ to match the IP recording. Real-time visualisation of the PNT signal was used to ensure stable and regular respiratory breathing patterns during the recording.

Before lung function measurements, the infants were clinically examined to exclude current respiratory infection. The infants were sedated with orally administered chloral hydrate $\left(50-100 \mathrm{mg} \cdot \mathrm{kg}^{-1}\right.$; maximum dose $1000 \mathrm{mg}$ ). All measurements were performed with commercial paediatric pulmonary function equipment (Babybody Masterscreen), as described previously [15, 16]. Functional residual capacity (FRC) and specific airway conductance $(s G$ aw) were assessed by the plethysmographic method, and the maximum flow at FRC ( $\left.V^{\prime} \operatorname{maxFRC}\right)$ was determined using the rapid thoracoabdominal compression technique [17]. The lung function data were converted to z-scores adjusting for weight, length and/or sex [18-20]. Z-scores below -1.96 in $s G$ aw or $V^{\prime}$ maxFRC were defined abnormal and indicative of reduced lung function. 
TABLE 1 Characteristics of the study infants with successful impedance pneumography measurement (All) and in the subgroup who underwent a bronchial challenge test with methacholine (MIB)

\begin{tabular}{|c|c|c|}
\hline & All & MIB \\
\hline Study children $\mathbf{n}$ & 44 & 20 \\
\hline Boys & $28(64)$ & $13(65)$ \\
\hline Gestational age weeks & $39(29-42)$ & $39(35-42)$ \\
\hline Birth weight kg & $3.2(0.9-5.2)$ & $3.3(2.4-5.2)$ \\
\hline Age months & $15.0(5.0-28.0)$ & $18.0(9.0-28.0)$ \\
\hline Weight kg & $10.9(6.2-15.6)$ & $11.0(8.6-13.8)$ \\
\hline Skin prick test positive & $7(16)$ & $4(20)$ \\
\hline Atopic eczema & $18(41)$ & $10(50)$ \\
\hline Parental smoking & $12(27)$ & $5(25)$ \\
\hline Parental asthma & $14(32)$ & $9(45)$ \\
\hline \multicolumn{3}{|l|}{ Dominant respiratory symptom } \\
\hline Cough & $22(50)$ & $12(60)$ \\
\hline Wheeze & $10(23)$ & $4(20)$ \\
\hline Dyspnea & $5(11)$ & $2(10)$ \\
\hline Other & $16(7)$ & $2(10)$ \\
\hline Physician-diagnosed wheeze & $25(57)$ & $10(50)$ \\
\hline \multicolumn{3}{|c|}{ Medication during the last 2 months } \\
\hline Bronchodilators & $40(91)$ & $18(90)$ \\
\hline Corticosteroids & 4 (9) & $2(10)$ \\
\hline FRC mL & $233(125-380)$ & $237(166-380)$ \\
\hline FRC z-score & $0.4(-2.0-6.5)$ & $0.6(-0.6-4.1)$ \\
\hline$s G_{a w} \mathrm{kPa}^{-1} \cdot \mathrm{s}^{-1}$ & $1.8(0.5-5.9)$ & $2.3(1.4-5.9)$ \\
\hline sGaw z-score & $-1.7(-5.7-8.9)$ & $-0.5(-2.6-8.9)$ \\
\hline$V^{\prime} \operatorname{maxFRC} \mathrm{mL} \cdot \mathrm{s}^{-1}$ & $189(18-465)$ & $260(122-465)$ \\
\hline$V^{\prime} \operatorname{maxFRC} z$-score & $-1.1(-3.7-1.4)$ & $-0.8(-2.0-1.4)$ \\
\hline
\end{tabular}

Data are presented as $\mathrm{n}(\%)$ or median (range). FRC: functional residual capacity; sGaw: specific airway conductance; V'maxFRC: maximal flow at FRC.

After baseline measurements of $V^{\prime}$ maxFRC, increasing doses of methacholine chloride were administered using an inhalation-synchronized dosimeter (Spira Electro 2, Spira Respiratory Care Centre Ltd, Hämeenlinna, Finland), as described previously $[15,16]$. The procedure was continued until a $40 \%$ decrease in $V^{\prime} \max F \mathrm{RC}$ was observed or the maximum dose of methacholine had been administered.

Impedance pneumography

In IP, the electrical impedance of the thorax is measured by feeding a small high-frequency current through one electrode pair and measuring the resulting voltage signal through another electrode pair. The electrical impedance increases as air enters the lungs during inspiration and decreases with expiration. The resulting volume-oriented signal can be differentiated to obtain a flow-rate signal [21]. No calibration of any sort was performed on the IP signal. The current-feeding electrodes were placed on both sides of the thorax on the mid-axillary line at the height of the fifth intercostal space and the voltage measurement electrodes were placed on the arms opposing the other electrode pair. The distortive impedance oscillations resulting from cardiac activity were removed by a filtering technique developed for this purpose [11]. IP and ECG signals were recorded and stored at a $256 \mathrm{~Hz}$ sampling rate by a small recording device (design of Tampere University of Technology, Finland) using normal Ag-AgCl ECG electrodes (Blue Sensor N, Ambu Ltd, Ballerup, Denmark). The IP signal was time-differentiated into a flow-related signal using a Savitzky-Golay filter of second order with window length of $50 \mathrm{~ms}$ [22].

\section{Tidal breathing indices}

The tidal airflow curve shape was estimated using the parameters time to peak tidal expiratory flow to total expiratory time ratio (TPTEF/TE) and volume to peak expiratory flow to total expiratory volume ratio $(V \mathrm{PTEF} / V \mathrm{E})$, as recommended [1]. From each 1-min recording, the parameters were determined by manually extracting a segment of regular breathing containing a median (range) of 26 (5-46) consecutive breaths. The TBFV parameters were derived from each breath and averaged for each recording with a trimmed mean that rejected the highest and lowest 5\% of values. Putatively, TPTEF/TE and VPTEF/VE should decrease as a result of bronchial obstruction (MIB), and increase after bronchodilation (BRD). 
Estimation of agreement between IP and PNT

The agreement between relative flow signals of IP (V'IP) and PNT (V'PNT) for each measurement was analysed as described previously [13]. A detailed description of the measures, i.e. the sample-by-sample difference (Dss), the average deviation of linearity (DL) and the largest single deviation (Dmax) are presented in the supplementary material. Briefly, both signals were first normalised in amplitude so that the maximal inspiratory flow equalled $100 \%$. Dss is the mean difference of each signal sample between normalised IP and PNT (lower is better). DL describes how much the signals deviate from a line that is fitted to a plot where both signals are drawn against each other (lower is better). The linearity is assessed at 10 different flow rates and DL and Dmax represent the mean and maximal deviation from linearity, respectively.

\section{Statistical analysis}

The repeatability of TBFV indices was determined using the paired recordings at the baseline. Within-subject standard deviation, SDws, was calculated for each patient for each of the baseline variables as $\mathrm{SD}_{\mathrm{WS}}=\left|\mathrm{X}_{\mathrm{BL} 1}-\mathrm{X}_{\mathrm{BL} 2}\right| / \sqrt{2}$, where $\mathrm{X}$ is the measured value obtained during measurements at BL1 and BL2, respectively. Bland-Altman plots including the coefficient of repeatability are included in the supplementary material.

A Pearson linear correlation was calculated to estimate the association of indices of agreement and TBFV with lung function at baseline conditions. A paired Wilcoxon signed rank test was used to compare changes during the different phases of the bronchial challenge test. Between-group comparisons were estimated using the Kruskal-Wallis analysis of variance, and post hoc comparisons with the Mann-Whitney U test.

\section{Results}

Successful IP recordings were obtained for 44 infants. The analysis of IP signal was precluded by motion artefacts $(n=2)$, poor wire connection $(n=1)$ or failure to use the IP device by the technician $(n=3)$. After lung function and clinical assessments, the infants were divided into four clinical groups: 1) 21 infants had normal lung function at baseline; 2) 11 infants had significant lower airway obstruction (LAO), indicated by low $s$ aw and $V^{\prime}$ maxFRC with a typical concave flow-volume curve [17]; 3) eight infants had signs of upper airway obstruction (UAO), indicated by low $s$ aw with inspiratory looping in pressure/flow tracings [23]; and 4) four infants had reduced lung function and radiographic findings of an interstitial lung disease (ILD) in CT scans. In the third group, with UAO, five infants had a structural abnormality that was verified on bronchoscopy (tracheal stenosis $(n=2)$, tracheo/laryngomalacia $(n=3)$ ).

A subsample of 20 infants underwent a bronchial challenge test. Exclusion from the challenge test was due to age under 6 months $(n=2)$, significant bronchial obstruction at baseline $(n=16)$, awakening $(n=1)$, or insufficient reproducibility of $V^{\prime} \operatorname{maxFRC}$ at baseline $(\mathrm{n}=5)$.

\section{Agreement between the methods}

Dss (SD) between the methods at the baseline measurements BL1 and BL2 were 5.5\% (1.6) and 5.5\% (1.6) of tidal peak inspiratory flow (TPIF), respectively. The average Dss (from BL1 and BL2) was significantly associated with $s$ aw $(\mathrm{p}=0.008)$ and $V^{\prime} \operatorname{maxFRC}(\mathrm{p}=0.009)$ expressed as $\mathrm{z}$-scores, but not with FRC $(\mathrm{p}=0.90)$. An abnormal finding in chest auscultation during lung function testing was associated with increased Dss $(\mathrm{p}=0.045)$, but a history of physician-confirmed wheeze $(\mathrm{p}=0.25)$ or prematurity $(\mathrm{p}=0.54)$ were not. DL $(\mathrm{SD})$ for BL1 and BL2 were 3.9\% (1.7) and 4.3\% (2.4) of TPIF, respectively. The average baseline DL was significantly associated with $s$ aw $(\mathrm{p}=0.002)$ and $V^{\prime} \operatorname{maxFRC}(\mathrm{p}=0.004)$, but not with FRC $(\mathrm{p}=0.88)$. An abnormal finding in chest auscultation was associated with increased DL $(p=0.021)$ but a history of physician-confirmed wheeze $(p=0.43)$ or prematurity $(p=0.58)$ were not.

The agreement between IP and PNT signals in different clinical groups is summarised in table 2 and in the supplementary material. Dmax and DL differed significantly between groups; in post hoc analyses, the agreement between IP and PNT was significantly poorer in infants with LAO (Dmax, $\mathrm{p}=0.001 ; \mathrm{DL}, \mathrm{p}<0.001$ ), compared to in infants with normal lung function. The UAO group differed significantly only in terms of $\mathrm{Dmax}(\mathrm{p}=0.028)$, whereas in the ILD group, the agreement was similar to that in infants with normal lung function.

\section{Tidal breathing parameters}

The average repeatability (SDws) of TBFV parameters at baseline was similar between PNT (0.025 for TPTEF/TE and 0.025 for VPTEF/VE) and IP ( 0.029 for TPTEF/TE and 0.030 for VPTEF/VE; $\mathrm{p}=0.24$ and p=0.38 between methods, respectively). Bland-Altman plots illustrating the agreement between TBFV parameters obtained from PNT and IP are presented in figure 1. Compared to PNT, IP slightly underestimated the parameters, the mean difference (95\% limits of agreement) between IP and PNT being $-0.025(-0.113-0.063)$ for TPTEF/TE and $-0.019(-0.112-0.073)$ for $V \mathrm{PTEF} / V \mathrm{E}$. 
TABLE 2 Agreement ( $\%$ of tidal peak inspiratory flow) between impedance pneumography and pneumotachograph signals at baseline in the four clinical groups of infants

\begin{tabular}{lcccc} 
& $\mathbf{n}$ & $\mathbf{D} \max$ & $\mathbf{D L}$ & Dss \\
\hline NLF & 21 & $-5.1(-14.6-7.6)$ & $3.2(1.1-6.6)$ & $4.7(3.8-7.1)$ \\
LAO & 11 & $-11.1(-26.6-1.6)$ & $5.2(3.1-9.7)$ & $5.6(3.8-10.6)$ \\
UAO & 8 & $-11.6(-31.3-4.8)$ & $4.7(2.1-12.3)$ & $5.2(4.1-8.5)$ \\
ILD & 4 & $-7.0(-20.4-0.45)$ & $3.4(1.1-12.3)$ & $5.6(4.2-7.0)$ \\
p-value & & 0.009 & 0.005 & 0.145 \\
\hline
\end{tabular}

Data are presented as median (range). The agreement was expressed as the sample-by-sample difference (Dss), the average deviation of linearity (DL), and the largest single deviation (Dmax) from linearity. Detailed descriptions of the measures are presented in the supplementary material. \#: p-values refer to the Kruskall-Wallis test. NLF: normal lung function; LAO: lower airway obstruction; UAO, upper airway obstruction; ILD: interstitial lung disease.

When using recordings with IP, the average $T \mathrm{PTEF} / T \mathrm{E}$ and $V \mathrm{PTEF} / V \mathrm{E}$ at baseline were significantly associated with $V^{\prime} \operatorname{maxFRC}(\mathrm{r}=0.40, \mathrm{p}=0.012$ and $\mathrm{r}=0.39, \mathrm{p}=0.013$, respectively) but not with $s$ aw $(\mathrm{p}=0.28$ and $\mathrm{p}=0.10$, respectively). The relationship with lung function was similar with PNT (figure 2).

\section{Bronchial challenge test}

The agreement between PNT and IP and the TBFV parameters in those infants who underwent the challenge test $(n=20)$ are presented by each phase in table 3. Compared to the mean of BL1 and BL2, no significant changes in agreement were observed during the MIB phase, but Dss increased during BRD phase $(\mathrm{p}=0.002)$

After the last dose of methacholine, $V^{\prime}$ maxFRC decreased significantly $(\mathrm{p}<0.001)$, and in 18 infants the change was $>40 \%$. Compared to baseline, there was an expected decrease in TPTEF/TE and VPTEF/VE during the MIB phase, but the change was statistically significant only in VPTEF/VE measured by PNT.

\section{Discussion}

In this study of symptomatic infants with a wide range of lung function impairments, TBFV profiles were consistent between IP and PNT in most subjects. Despite the fact that the modes of measurement represent completely different principles, the mean differences were minor. No calibration was required on IP in this study because the derived TBFV parameters do not depend on absolute measures of flow. However, for deriving complete TBFV curves by IP, high linearity with the flow signal is required. In preschool children, we have previously confirmed the validity of IP for recording tidal airflow profiles [13], and found that tidal breathing variability assessed by IP during home recordings is related to childhood asthma risk [14]. We have now found the linearity of IP to be comparable and therefore satisfactory to also study similar clinical correlates in infants. However, in subgroups of infants, the agreement was significantly affected by lower and upper airway obstruction. This may be reflected in TBFV parameters so that the results are not interchangeable between PNT and IP.
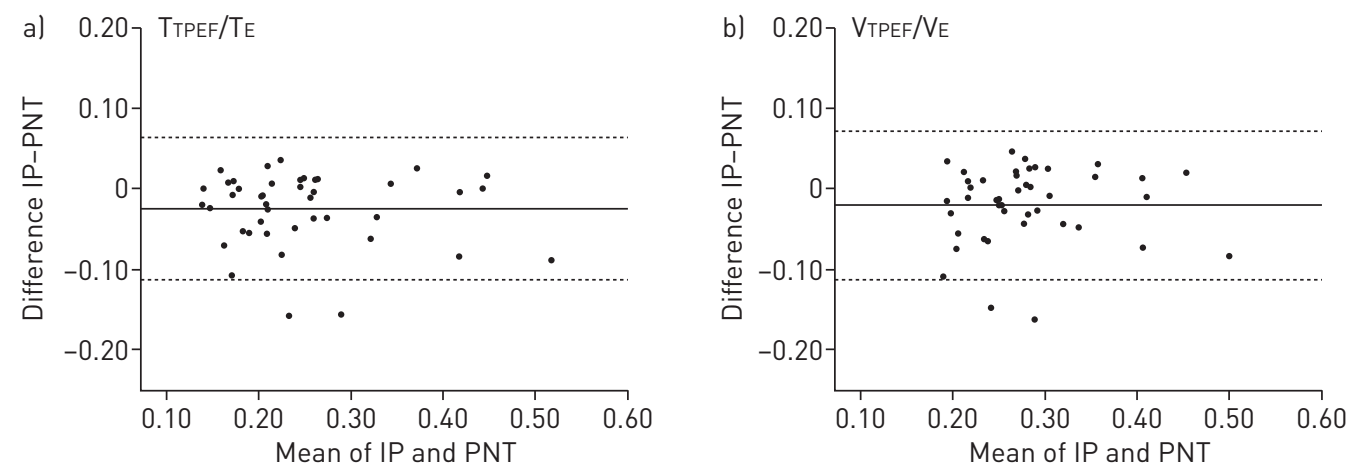

FIGURE 1 Bland-Altman comparison plot of the tidal breathing parameters a) ratio of time to peak tidal expiratory flow to total expiratory time (TPTEF/TE) and b) ratio of volume to peak expiratory flow to total expiratory volume (VPTEF/VE) obtained simultaneously with impedance pneumography (IP) and pneumotachography (PNT). The solid line denotes the mean; the dashed lines denote the $95 \%$ confidence intervals of the difference between the IP and PNT results. 
a)

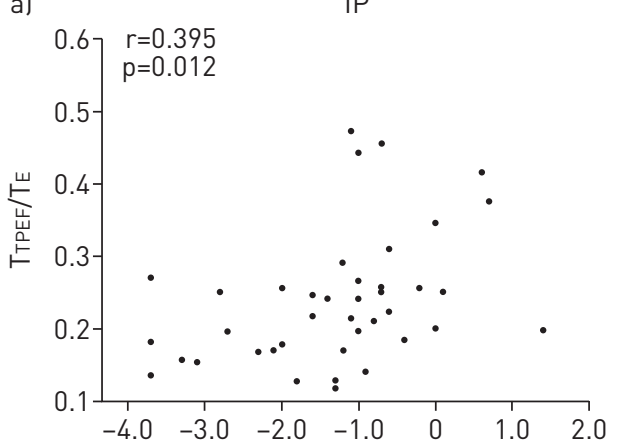

c)

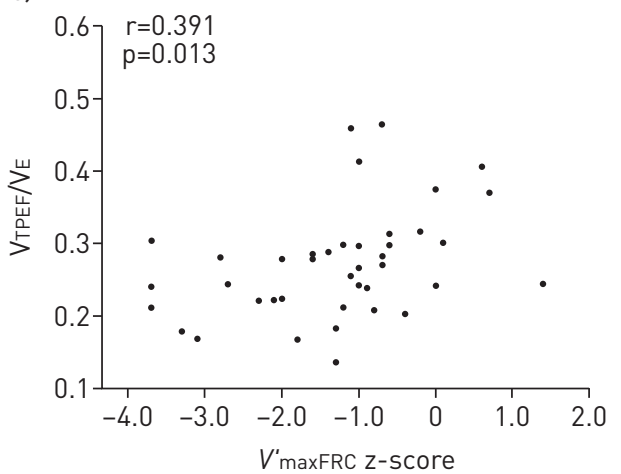

b)

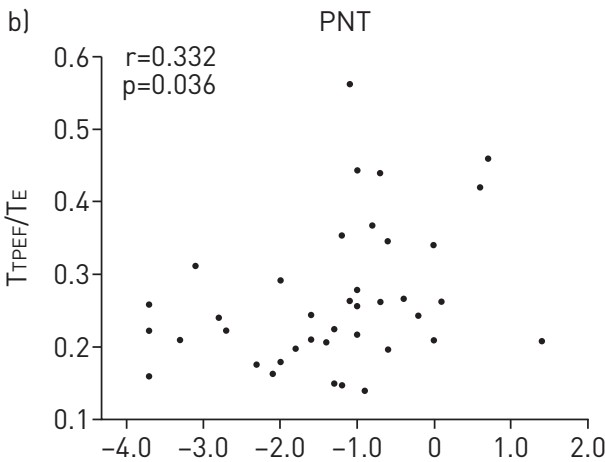

d)

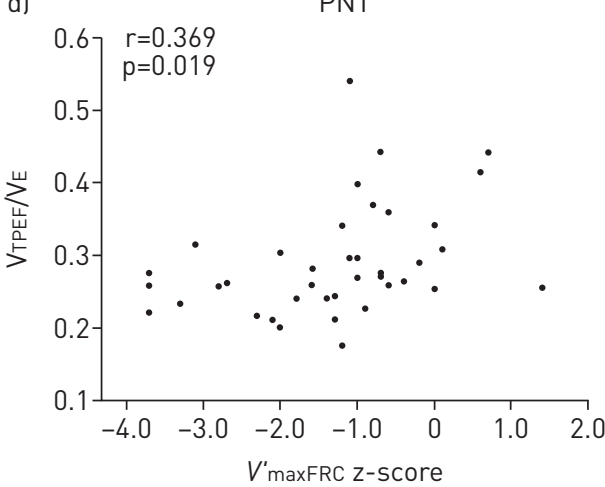

FIGURE 2 The association between the maximal flow at functional residual capacity (FRC) ( $V^{\prime}$ maxFRC) measured with the rapid thoracoabdominal compression technique, and the tidal breathing flow volume parameters $a, b)$ TPTEF/TE and $c, d)$ VPTEF/VE, obtained with impedance pneumography (IP, a and c) or pneumotachography (PNT, b and d).

Assessment of tidal breathing, including flow and volume, is usually accomplished by direct measurement at the airway opening using PNT [1,7]. Indirect, noninvasive techniques have the advantage of avoiding the mask or mouthpiece interfering with the breathing pattern $[24,25]$ and potentially also enabling long-term measurements of tidal breathing. In infants, respiratory inductance plethysmography (RIP) measures tidal breathing noninvasively [26], but its accuracy has been questioned unless calibrated using a PNT [27]. Two reports $[27,28]$ have suggested that the tidal breathing parameter TPTEF/TE is underestimated by RIP in

TABLE 3 Lung function in terms of the maximum flow at functional residual capacity, the agreement between flow signals derived from impedance pneumography and pneumotachography, and tidal breathing flow volume parameters (TPTEF/TE and VPTEF/VE) determined by the same at baseline, during methacholine-induced bronchoconstriction and after bronchodilator inhalation in the subgroup of infants in the bronchial challenge test $(n=20)$

\begin{tabular}{lccc} 
& BL & MIB & BRD \\
\hline $\begin{array}{l}\boldsymbol{V}^{\prime} \text { maxFRc mL } \\
\text { Agreement }\end{array}$ & $264(82)$ & $129(64)^{*}$ & $234(96)$ \\
DsS (\% of TPIF) & $4.8(0.8)$ & $5.4(1.2)$ & $6.2(1.9)^{* *}$ \\
D (\% of TPIF) & $3.2(1.1)$ & $3.3(1.1)$ & $3.8(1.6)$ \\
Tidal parameters & & & \\
$\quad T$ PTEF/TE (IP) & $0.28(0.09)$ & $0.24(0.09)$ & $0.24(0.07)$ \\
TPTEF/TE (PNT) & $0.30(1.1)$ & $0.26(0.07)$ & $0.27(0.09)$ \\
VPTEF/VE (IP) & $0.30(0.08)$ & $0.27(0.09)$ & $0.28(0.06)$ \\
VPTEF/VE (PNT) & $0.32(0.09)$ & $0.28(0.05)^{*}$ & $0.30(0.07)$
\end{tabular}

Results are expressed as mean (sD). The agreement was expressed as the sample-by-sample difference (Dss), and the average deviation of linearity (DL). BL: baseline; MIB, methacholine-induced bronchoconstriction; BRD: after bronchodilator inhalation; V'maxFRC: the maximum flow at functional residual capacity; TPIF: tidal peak inspiratory flow; TPTEF/TE: time to peak tidal expiratory flow to total expiratory time ratio; VPTEF/VE: volume to peak tidal expiratory flow to total expiratory volume ratio; IP: impedance pneumography; PNT: pneumotachography; ${ }^{*}: p<0.05 ;{ }^{* *}: p<0.01$ compared to baseline. 
small children. Our observation was similar for IP, which produced variably lower TPTEF/TE and VPTEF/VE values compared to PNT. In addition, symptomatic infants demonstrated an association of TBFV parameters with baseline lung function assessed with forced expirations. In this respect, both recording techniques (IP and PNT) showed a modest value as indices of lung function.

Three studies have previously investigated TBFV parameters during bronchial provocation tests in infants [29-31]. BENOIST et al. [29] described a concave flow volume curve in most infants after a final dose of methacholine, and a reduction in TPTEF/TE that was almost similar in sensitivity to that of $V^{\prime} \operatorname{maxFRC}$. In contrast, Aston et al. [30] and FREY et al. [31] found no change in TPTEF/TE after bronchial challenge with histamine, which is closer to our findings that show only minor changes in TPTEF/TE and VPTEF/VE during MIB. This weak relationship with acute changes in lung function could reflect the complex interactions of the elastic and flow-resistive properties of the lungs and neural control of breathing on TBFV parameters [32, 33].

The study sample represents infants undergoing assessment in a tertiary clinic due to severe lung symptoms, and is thereby heterogeneous and potentially biased. Owing to the small number of subjects in the subgroups, we cannot but speculate on the possible mechanisms of the poorer performance of IP in some infants. Interestingly, the agreement of IP was not significantly affected by MIB. This suggests that nonlinearities of IP are not related to changes in airway diameter per se but that more complex mechanisms are involved in infants with diseased lungs. The IP signal, which gives only a relative measure of volume change, is dependent on the aeration of the lung regions within the measurement sensitivity field formed in the thorax by the electrodes. Increased ventilation heterogeneity due to lung disease is a potential contributor to measurement errors. Asynchrony of chest wall motion is a typical feature in infants with upper and lower airway obstruction, due to altered pleural pressure swings acting on the compliant chest wall [34]. Potentially, asynchrony in the filling and emptying of the apical and basal parts of the lungs could lead to nonlinearity of the IP signal, compared to flow at the mouth. In contrast, we did not find any association between the measures of agreement and hyperinflation [35] expressed as high FRC level. Electrical conductivity of the thorax could be also affected by changes in venous return and blood volume during the breathing cycle [36], an effect that could be enhanced by the large pressure swings in infants with increased breathing work. Whether sedation also interferes with the IP signal is not so far known.

As with any instrument, PNT systems have limited accuracy and linearity, which is especially noteworthy in infants with small flow rates. Although care was taken to seal the mask, small leakages during recordings could also have contributed to nonlinearity between PNT and IP. It is also widely accepted that infant lung function techniques we utilised in this study have several limitations [37] and reflect different pathophysiological aspects of respiratory function. Therefore their modest associations with TBFV parameters were not unexpected.

With regards clinical implications, the results suggest that whereas IP seems applicable for most infants, the accuracy of IP may not be ideal for the measurement of TBFV profiles in infants with clinical signs of severe obstruction, chest wall asynchrony or abnormal lung auscultation, and their results should therefore be interpreted with caution. On the other hand, the agreement of tidal flow profiles seemed satisfactory in those infants with episodic respiratory symptoms but who were stable and clinically without signs of bronchial obstruction at the time of study. This group of infants could be in need of diagnostic evaluation, which is only possible by long-term monitoring of lung function. Recordings of longer duration also enable the calculation of more robust measures of tidal airflow profiles than the conventional TBFV parameters, as well as complex indices of flow variability [38]. By using nocturnal recordings with IP, we demonstrated that preschool children with a high risk of asthma present with an increased variation of tidal flow profile shape, and momentarily lowered chaoticity, compared to children with a lower risk of asthma [14]. Similar ambulatory applications could be envisioned for infants, but so far no data are available.

We conclude that IP is suited for the assessment of tidal flow profiles in most infants, but caution should be exercised when applied to subjects with clinically evident airway obstruction. The TBFV parameters derived are not interchangeable with those obtained by direct PNT but show a similar association with the lung function of the infant. Further investigations are needed to evaluate the applicability of IP in the long-term monitoring of lung function in infants.

\section{Acknowledgements}

We would like to thank Anssi Koivuselkä (Skin and Allergy Hospital, Helsinki, Finland) for practical work with the children.

\section{References}

1 Bates JHT, Schmalisch G, Filbrun D, et al. Tidal breath analysis for infant pulmonary function testing. Eur Respir J 2000; 16: 1180-1192.

2 Ranganathan S, Goetz I, Hoo A-F, et al. Assessment of tidal breathing parameters in infants with cystic fibrosis. Eur Respir J 2003; 22: 761-766. 
3 Schmalisch G, Wilitzki S, Wauer RR. Differences in tidal breathing between infants with chronic lung diseases and healthy controls. BMC Pediatr 2005; 5: 36.

4 Carlsen K-H, Lødrup Carlsen KC. Tidal breathing analysis and response to salbutamol in awake young children with and without asthma. Eur Respir J 1994; 7: 2154-2159.

5 Lødrup Carlsen KC, Stenzler A, Carlsen K-H. Determinants of tidal flow volume loop indices in neonates and children with and without asthma. Pediatr Pulmonol 1997; 24: 391-396.

6 Håland G, Lodrup Carlsen KC, et al. Reduced lung function at birth and the risk of asthma at 10 yrs of age. N Engl J Med 2006; 355: 1682-1689.

7 van der Ent CK, Breckel HJL, Mulder P, et al. Improvement of tidal breathing pattern analysis in children with asthma by on-line automatic data processing. Eur Respir J 1996; 9: 1306-1313.

8 Brown K, Aun C, Jackson E, et al. Validation of respiratory inductive plethysmography using the qualitative diagnostic calibration method in anesthetized infants. Eur Respir J 1998; 12: 935-943.

9 Baker LE, Geddes LA, Hoff HE. A comparison of linear and non-linear characterizations of impedance spirometry data. Med Biol Eng 1966; 4: 371-379.

10 Kurth F, Zinnow F, Prakapenia A, et al. Continuous non-invasive monitoring of tidal volumes by measurement of tidal impedance in neonatal piglets. PLoS One 2011; 6: e21003.

11 Seppä V-P, Hyttinen J, Viik J. A method for suppressing cardiogenic oscillations in impedance pneumography. Physiol Meas 2011; 32: 337-345.

12 Seppä V-P, Hyttinen J, Uitto M, et al. Novel electrode configuration for highly linear impedance pneumgraphy. Biomed Tech (Berl) 2013; 58: 35-38.

13 Seppä V-P, Pelkonen AS, Kotaniemi-Syrjänen A, et al. Tidal breathing flow measurement in awake young children by using impedance pneumography. J Appl Physiol 2013; 115: 1725-1731.

14 Seppä V-P, Pelkonen A, Kotaniemi-Syrjänen A, et al. Tidal flow variability measured by impedance pneumography relates to childhood asthma risk. Eur Respir J 2016; 47: 1687-1696.

15 Kotaniemi-Syrjänen A, Malmberg LP, Pelkonen A, et al. Airway responsiveness: associated features in infants with recurrent respiratory symptoms. Eur Respir J 2007; 30: 1150-1157.

16 Malmberg LP, von Wright L, Kotaniemi-Syrjänen A, et al. Methacholine-induced lung function changes measured with infant body plethysmography. Pediatr Pulmonol 2010; 46: 362-368.

17 Sly PD, Tepper RS, Henschen M, et al. ERS/ATS Task Force on Standards for Infant Respiratory Function Testing. Eur Resp J 2000; 16: 741-748.

18 Hülskamp G, Hoo A-F, Ljungberg $\mathrm{H}$, et al. Progressive decline in plethysmographic lung volumes in infants: physilogy or technology? Am J Respir Crit Care Med 2003; 168: 1003-1009.

19 Kraemer R, Graf Bigler U, Casaulta Aebischer C, et al. Clinical and physiological improvement after inhalation of low-dose beclomethasone dipropionate and salbutamol in wheezy infants. Respiration 1997; 64: 342-349.

20 Hoo A-F, Dezateux C, Hanrahan JP, et al. Sex-specific prediction equations for V'maxFRC in infancy. Am J Respir Crit Care Med 2002; 165: 1085-1092.

21 Seppä V-P, Viik J, Hyttinen J. Assessment of pulmonary flow using impedance pneumography. IEEE Trans Biomed Eng 2010; 57: 2277-2285.

22 Savitzky A, Golay MJE. Smoothing and differentiation of data by simplified least squares procedures. Anal Chem 1964; 36: 1627-1639.

23 Stocks J, Thomson A, Wong C. Pressure-flow curves in infancy. Pediatr Pulmonol 1985; 1: 33-40.

24 Dolfin T, Duffty P, Wilkes D, et al. Effects of a face mask and pneumotachnograph on breathing in sleeping infants. Am Rev Respir Dis 1983; 128: 977-979.

25 Schmalisch G, Foitzik B, Wauer RR, et al. Effect of apparatus dead space on breathing parameters in newborns: "flow-through" versus conventional techniques. Eur Respir J 2001; 17: 108-114.

26 Stick S, Ellis E, LeSouëf $\mathrm{P}$, et al. Validation of respiratory inductance pletysmography (Respitrace) for measurements of tidal breathing parameters in newborns. Pediatr Pulmonol 1992; 14: 187-191.

27 Jackson E, Stocks J, Pilgrim I, et al. A critical assessment for the measurement of uncalibrated respiratory inductance plethysmography (Respitrace) for the measurement of tidal breathing parameters in newborns and infants. Pediatr Pulmonol 1995; 20: 119-124.

28 Manczur T, Greenough A, Hooper R, et al. Tidal breathing parameters in young children: comparison of measurement by respiratory inductance plethysmography to a facemask pneumotachograph system. Pediatr Pulmonol 1999; 28: 436-441.

29 Benoist MR, Brouard JJ, Rufin P, et al. Ability of new lung function tests to assess methacholine-induced airway obstruction in infants. Pediatr Pulmonol 1994; 18: 308-316.

30 Aston $\mathrm{H}$, Clarke J, Silverman M. Are tidal breathing indices useful in infant bronchial challenge tests? Pediatr Pulmonol 1994; 17: 225-230.

31 Frey U, Silverman M, Suki B. Analysis of harmonic content of the tidal flow waveforms in infants. J Appl Physiol 2001; 91: 1687-1693.

32 Seddon PC, Davis GM, Coates AL. Do tidal expiratory flow patterns reflect lung mechanics in infants? Am J Respir Crit Care Med 1996; 153: 1248-1252.

33 van der Ent CK, van der Grinten CP, Meessen NE, et al. Time to peak expiratory flow and the neuromuscular control of expiration. Eur Respir J 1998; 12: 646-652.

34 Allen JL, Wolfson MR, McDowell $\mathrm{K}$, et al. Thoracoabdominal asynchrony in infants with airflow obstruction. Am Rev Respir Dis 1990; 141: 337-342.

35 Kraemer R, Frey U, Wirz-Sommer C, et al. Short-term effect of albuterol, delivered via a new auxiliary device in wheezy infants. Am Rev Respir Dis 1991; 144: 347-351.

36 Brecher GA, Hubay CA. Pulmonary blood flow and venous return during spontaneous respiration. Circulation 1955; 111: 210-214.

37 England S. Current techniques for assessing pulmonary function in the newborn and infant: advantages and limitations. Pediatr Pulmonol 1988; 4: 48-53.

38 Veiga J, Lopes AJ, Jansen JM, et al. Airflow pattern complexity and airway obstruction in asthma. J Appl Physiol 2011; 111: 412-419. 